\title{
Correction to: CT and clinical assessment in asymptomatic and pre-symptomatic patients with early SARS-CoV-2 in outbreak settings
}

\author{
Nicole Varble ${ }^{1,2} \cdot$ Maxime Blain $^{1} \cdot$ Michael Kassin $^{1,3} \cdot$ Sheng Xu ${ }^{1} \cdot$ Evrim B. Turkbey ${ }^{3} \cdot$ Amel Amalou $^{1} \cdot$ Dilara Long $^{1} \cdot$

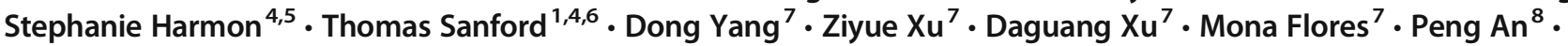 \\ Gianpaolo Carrafiello ${ }^{9,10}$ - Hirofumi Obinata ${ }^{11}$ - Hitoshi Mori ${ }^{11}$ - Kaku Tamura ${ }^{11}$ - Ashkan A. Malayeri ${ }^{3}$. \\ Steven M. Holland ${ }^{12} \cdot$ Tara Palmore $^{13} \cdot$ Kaiyuan Sun ${ }^{14} \cdot$ Baris Turkbey $^{3,4} \cdot$ Bradford J. Wood $^{1,3,4,15}$
}

Published online: 8 December 2020

(C) European Society of Radiology 2020

\section{Correction to: European Radiology} https://doi.org/10.1007/s00330-020-07401-8

The original version of this article, published on 04 November 2020, unfortunately contained a mistake. The following correction has therefore been made in the original:

The last sentence of section "Characterization of CT infiltrates in asymptomatic patients" was worded incorrectly. The correct wording is: "The average attenuation of the

The online version of the original article can be found at https://doi.org/ $10.1007 / \mathrm{s} 00330-020-07401-8$

Bradford J. Wood

bwood@nih.gov

1 Center for Interventional Oncology, Radiology and Imaging Sciences, NIH Clinical Center and National Cancer Institute, Center for Cancer Research, National Institutes of Health, Bethesda, MD, USA

2 Philips Research North America, Cambridge, MA, USA

3 Department of Radiology and Imaging Sciences, National Institutes of Health, Bethesda, MD, USA

4 National Cancer Institute, National Institutes of Health, Bethesda, MD, USA

5 Clinical Research Directorate, Frederick National Laboratory for Cancer Research sponsored by the National Cancer Institute, Frederick, MD, USA

6 State University of New York Upstate Medical University, Syracuse, NY, USA

7 Nvidia Corporation, Bethesda, MD, USA infiltrates was - $610 \pm 94 \mathrm{HU}$, while nondiseased lung had an average attenuation of - $830 \pm 42$ HU."

The original article has been corrected.

Publisher's note Springer Nature remains neutral with regard to jurisdictional claims in published maps and institutional affiliations.
8 Department of Radiology, Xiangyang NO. 1 People's Hospital Affiliated to Hubei University of Medicine, Xiangyang, Hubei, China

9 Department of Radiology, Fondazione IRCCS Cà Granda Ospedale Maggiore Policlinico, Milan, Italy

10 Department of Health Sciences, University of Milano, Milan, Italy

11 Self-Defense Forces Central Hospital, Tokyo, Japan

12 National Institute of Allergy and Infectious Diseases, National Institutes of Health, Bethesda, MD, USA

13 Hospital Epidemiology Service, NIH Clinical Center, Bethesda, MD, USA

14 Division of International Epidemiology and Population Studies, Fogarty International Center, National Institutes of Health, Bethesda, MD, USA

15 National Institute of Biomedical Imaging and Bioengineering, Bethesda, MD, USA 